\title{
Some Remarks on the KP System of the Camassa-Holm Hierarchy ${ }^{\star}$
}

\author{
Giovanni ORTENZI $\dagger^{\dagger^{1} \dagger^{2}}$ \\ $\dagger^{1}$ Dipartimento di Matematica Politecnico di Torino, \\ Corso Duca degli Abruzzi 24, 10129 Torino, Italy \\ $\dagger^{2}$ Dipartimento di Matematica e Applicazioni Università di Milano Bicocca, \\ Via R. Cozzi 53, 20125 Milano, Italy \\ E-mail: giovanni.ortenzi@unimib.it
}

Received October 31, 2006, in final form January 22, 2007; Published online March 13, 2007

Original article is available at http://www.emis.de/journals/SIGMA/2007/047/

\begin{abstract}
We study a Kadomtsev-Petviashvili system for the local Camassa-Holm hierarchy obtaining a candidate to the Baker-Akhiezer function for its first reduction generalizing the local Camassa-Holm. We focus our attention on the differences with the standard $\mathrm{KdV}-\mathrm{KP}$ case.
\end{abstract}

Key words: KP hierarchy; CH hierarchy; Sato Grassmannian

2000 Mathematics Subject Classification: 37K10; 35Q53

\section{Introduction}

The Camassa-Holm $(\mathrm{CH})$ equation with zero critical velocity [2]

$$
u_{t}-u_{x x t}=6 u_{x} u-4 u_{x x} u_{x}-2 u u_{x x x}
$$

and his multi fields extensions $[1,4,12,14,15,20,21,22,26]$ are widely studied integrable systems because they show a number of properties different from the standard Gelfand-Dikii systems such as Korteweg-de Vries (KdV). Its study is important to a deep understanding of infinite-dimensional integrability.

One of the most important differences between $\mathrm{CH}$ and $\mathrm{KdV}$ is that, even if $\mathrm{CH}$ has the same dispersionless part of the $\mathrm{KdV}$ equation

$$
u_{t}=-u_{x x x}+6 u u_{x}
$$

it does not admit tau structure in the sense of Dubrovin's classification scheme [10]. Classically, for the Kadomtsev-Petviashivili (KP) equation and its reductions, the tau structure is related to the fact that the Baker-Akhiezer function $\psi$ of the hierarchy satisfies a bilinear relation $[8$, $27,28]$.

The bi-Hamiltonian method allows the interpretation of the KP system as a generalization of the conservation laws for the corresponding Noether currents. This construction [13] starts from the Riccati equation related to the system. In the KdV case the equation

$$
h_{x}+h^{2}=u+z^{2}, \quad \text { with } \quad h(z):=z+\sum_{i \geq-1} \frac{h_{i}}{z^{i}},
$$

${ }^{\star}$ This paper is a contribution to the Proceedings of the Workshop on Geometric Aspects of Integrable Systems (July 17-19, 2006, University of Coimbra, Portugal). The full collection is available at http://www.emis.de/journals/SIGMA/Coimbra2006.html 
is the relation satisfied by the conserved densities $h_{i}$ of $\mathrm{KdV}$. Using the Noether theorem one can associate to every conserved density $h_{i}$ a current $H_{i}^{(3)}$ such that $\partial_{t} h_{i}=\partial_{x} H_{i}^{(3)}$. Every commuting symmetry in the KdV hierarchy is related to a different current and then, for a symmetry of $\mathrm{KdV}$ involving the time $\partial / \partial t_{s}=\partial_{s}$, it holds $\partial_{s} h_{i}=\partial_{x} H_{i}^{(s)}$. Using the generator $h(z)$ we can collect the currents into a set of generators of currents satisfying

$$
\partial_{s} h(z)=\partial_{x} H^{(s)}(z) .
$$

At this stage this set of equations is simply a way to rewrite the equations of the KdV hierarchy.

The amazing property of the currents $H^{(s)}$ is that they belong to a very particular space called $H_{+}$. This is the space generated by the linear span on $C^{\infty}\left(S^{1}, \mathbb{R}\right)$ of the Faà di Bruno polynomials $h^{(n)}:=\left(\partial_{x}+h\right)^{n} \cdot 1$ of the conserved density $h$. There is a unique way to write every current $H^{(s)}$ as $H^{(s)}(x, z)=\sum_{i=0}^{s} c_{i}(x) h^{(i)}(x, z)$.

Without account of (1), the equation (2) becomes an infinite differential system in an infinite number of fields. This is equivalent to the KP equation presented by the Japanese school. This system is a linear flow on a suitable Grassmannian whose $H_{+}$is the positive part. A key property [24] of $H_{+}$for the relation between KP and Grassmannian flows is that this space is invariant under the action of the operators $\left(\partial_{s}+H^{(s)}\right)$ :

$$
\left(\partial_{s}+H^{(s)}\right) H_{+} \subset H_{+}
$$

Thanks to (3) the KP system can be written as

$$
\left(\partial_{s}+H^{(s)}\right) H^{(r)}=H^{(s+r)}+\sum_{i=1}^{s} H_{i}^{r} H^{(s-i)}+\sum_{i=1}^{r} H_{i}^{s} H^{(r-i)} .
$$

If we forget the Faà di Bruno rule for construction of the space $H_{+}$, the currents $H^{(s)}$ are simply Laurent series in $z$ and a collection $\left\{H^{(s)}\right\}_{s \geq 0}$ is related ([13]) to a point of the positive part $H_{+}$ of the Sato Grassmannian $\mathcal{W}$ defined e.g. in [28]. The equation (4) describes now a flow on $\mathcal{W}$ which admits as reduction the KP system imposing the relation between the currents and the Faà di Bruno polynomials. It is called the central system for KP. The time $t_{1}$ of the central system can be identified with the variable $x$ of KP because the conservation of the linear momentum of KP equation.

This construction is a hint for the existence of bilinear relations [13] and one wonders whether the same scheme can be applied to generalizations of $\mathrm{CH}$ hierarchy.

The study of KP equation for $\mathrm{CH}$ is interesting in this direction.

In [3] the authors show the existence of a KP equation constructed starting from local symmetries of the $\mathrm{CH}$ equation. In this paper we plan to pursue these ideas further. After a review of the results presented in [3], we study the KP-CH system obtaining a candidate for the BakerAkhiezer function of the integrable reductions of the system. Next we consider the problem of the reduction from KP-CH to the local Camassa-Holm hierarchy. By means of this process we obtain a 3 -field integrable system which generalizes the local symmetries for $\mathrm{CH}$.

\section{The full CH hierarchy}

It was known $[2,16]$ that $\mathrm{CH}$ is bi-Hamiltonian on the vector space $C^{\infty}\left(S^{1}, \mathbb{R}\right)$ by means of the Poisson pencil

$$
P_{z^{2}}=P_{0}-z^{2} P_{1}=\frac{1}{2} \partial_{x}-\frac{1}{2} \partial_{x}^{3}-z^{2}\left(-m \partial_{x}-\partial_{x} m\right), \quad z \in \mathbb{R},
$$


where $m=\left(1-\partial_{x}^{2}\right) u$ and we call $z^{2}$ the pencil parameter for later convenience. The theory of bi-Hamiltonian systems (see e.g. [23]) shows that the conserved quantities for $\mathrm{CH}$ are the coefficients of the potential of an exact 1-form $v$ in the kernel of the Poisson pencil. If $H(z)=$ $\sum_{k=-\infty}^{+\infty} H_{k} z^{-k}$ satisfy $P_{z^{2}} d H(z)=0$ then one can construct the Lenard-Magri recursion $P_{0} d H_{k}=$ $P_{1} d H_{k+2}$. This chain implies that all $H_{k}$ are in involution w.r.t. both the Poisson pencils.

Every element $v(z)$ in $\operatorname{Ker} P_{z^{2}}$ satisfies the equation:

$$
\frac{1}{4} v^{2}-\frac{1}{2} v_{x x} v+\frac{1}{4} v_{x}^{2}+z^{2} m v^{2}=f(m, z),
$$

where $f(m, z)$ satisfies $f_{x}=0$. It turns out that $v$ is an exact 1-form if $f$ does not depend on $m$. Without loss of generality, we can put $f(z)=z^{2} / 4$.

The equation (5) can be solved iteratively developing $v$ in $z$. In order to find the potential $H(z)$ we evaluate $v$ on the generic vector $\dot{m}$ :

$$
\begin{aligned}
\langle v, \dot{m}\rangle & \stackrel{(5)}{=} \int_{S^{1}} v \frac{d}{d t}\left(\frac{z^{2}}{4 v^{2}}+\frac{1}{2 z^{2}} \frac{v_{x x}}{v}-\frac{1}{4 z^{2}} \frac{v_{x}^{2}}{v^{2}}-\frac{1}{4 z^{2}}\right) d x \\
& =\frac{d}{d t} \int_{S^{1}} \frac{1}{2 v} d x+\int_{S^{1}} \partial_{x}\left(\dot{v_{x}}+\frac{v_{x} \dot{v}}{v}\right) d x=\frac{d}{d t} \int_{S^{1}} \frac{1}{2 v} d x
\end{aligned}
$$

Therefore $H(z)=\int_{S^{1}}(1 / 2 v) d x$ is a generator of conserved quantities.

Let us focus now our attention on the density $h$ of $H$. It is obviously defined up to a normalization and up to a total derivative. Paying attention to these 'gauge' choices one can put

$$
h=z \frac{1}{2 v}+\partial_{x}(\ln \sqrt{v})
$$

and then the equation (5) becomes

$$
h_{x}+h^{2}=\frac{1}{4}+z^{2} m
$$

By construction, all the solutions of this equation define conserved quantities for $\mathrm{CH}([3,19,25]$ and, in the context of the inverse spectral problem, $[5,7])$.

The possible solutions of (6) are two functions, depending on the essential singularity point

of $h(z)$. The first one, $h(z)=\sum_{i=-1}^{+\infty} h_{i} z^{-i}$, has its essential singularity in 0 . It corresponds to the Lenard-Magri recursion starting from the Casimir of $P_{1}$ and all the quantities are local in $m$.

The second one, $k(z)=\sum_{i=0}^{+\infty} k_{i} z^{i}$, contains $\mathrm{CH}$ and it has an essential singularity at infinity. It corresponds to the Lenard-Magri recursion starting from the Casimir of $P_{0}$, and it has an infinite number of quantities nonlocal in $m$.

The calculation of these solutions is not difficult but a little bit involved. It is performed explicitly in [3]. We now simply list and comment the results.

\section{The local hierarchy}

The local hierarchy is related to the solutions of (6) of the form $h(z)=h_{-1} z+h_{0}+h_{1} / z+$ $h_{2} / z^{2}+\cdots$ :

$$
h_{-1}=\sqrt{m}, \quad h_{0}=\left(\ln \left(m^{-1 / 4}\right)\right)_{x},
$$




$$
\begin{aligned}
h_{1} & =\frac{1}{8 \sqrt{m}}-\frac{1}{8} \frac{m_{x x}}{\sqrt{m^{3}}}+\frac{5}{32} \frac{m_{x}^{2}}{\sqrt{m^{5}}}, \\
h_{2} & =\left(-\frac{1}{16 m}+\frac{1}{16} \frac{m_{x x}}{m^{2}}+\frac{5}{64} \frac{m_{x}^{2}}{m^{3}}\right)_{x}, \quad \ldots
\end{aligned}
$$

The conserved quantities $H_{i}=\int h_{i-1} d x$ for $i=0,1,2, \ldots$ are nontrivial only when $h_{i-1}$ are not a total derivative, i.e. when $i$ is even. Using only the even conserved quantities we obtain $H\left(z^{2}\right)=\sum_{i \geq 0} H_{2 i} z^{-2 i}$

We can iterate the Lenard-Magri chain defining an infinite number of vector fields $X_{2 i}=$ $P_{0} d H_{2 i}=P_{1} d H_{2 i+2}$ pairwise commuting and we identify $X_{2 i}=\partial_{2 i} m$. The first one, already presented in [2], is the local Camassa-Holm equation

$$
\partial_{0} m=\left(\partial_{x}-\partial_{x}^{3}\right) \frac{1}{4 \sqrt{m}}
$$

This part of the hierarchy generates the negative flows of $\mathrm{CH}$.

\section{The nonlocal hierarchy}

The nonlocal hierarchy is related to the solutions of (6) of the form $k(z)=k_{0}+k_{-1} z+k_{-2} z^{2}+\cdots$ :

$$
\begin{aligned}
k_{0}= & \frac{1}{2} \\
k_{-1} & =0 \\
k_{-2} & =\left(1+\partial_{x}\right)^{-1} m=u-u_{x} \\
k_{-3} & =0 \\
k_{-4} & =-\left(1+\partial_{x}\right)^{-1}\left(\left(1+\partial_{x}\right)^{-1} m\right)^{2}=-u^{2}-u_{x}^{2}+\text { total derivatives, } \\
k_{-5} & =0 \\
k_{-6} & =2\left(1+\partial_{x}\right)^{-1}\left(\left(1+\partial_{x}\right)^{-1}\left(\left(1+\partial_{x}\right)^{-1} m\right)^{2}\left(1+\partial_{x}\right)^{-1} m\right) \\
& =u^{3}+u u_{x}^{2}+\text { total derivatives, } \quad \ldots
\end{aligned}
$$

We remark that one can continue the iteration thanks to the invertibility of the operator $1+\partial_{x}$ in the space of smooth periodic functions [3]. The densities are increasingly non-local in $m$, but the first three are local in the field $u=\left(1-\partial_{x}^{2}\right)^{-1} m$.

As in the local case one can define conserved quantities $K_{-i}=\int k_{-i} d x$ for $i=0,1,2, \ldots$ Taking only the nontrivial ones, $i=2,4,6, \ldots$, we obtain $K\left(z^{2}\right)=\sum_{i \geq 1} K_{-2 i} z^{2 i}$.

We can iterate the Lenard-Magri chain defining an infinite number of vector fields $Y_{-2 i}=$ $P_{0} d K_{-2 i}=P_{1} d K_{-2 i+2}:=\partial_{-2 i} m$ pairwise commuting. The first nontrivial vector field is the conservation of the linear momentum $\partial_{-2} m=P_{1} d K_{-2}=P_{0} d K_{-4}=-m_{x}$. The second one is the standard Camassa-Holm equation with null critical velocity

$$
\partial_{-4} m=P_{1} d K_{-4}=-\left(m \partial_{x}+\partial_{x} m\right)\left(1-\partial_{x}^{2}\right)^{-1}\left(-2 u+2 u_{x x}\right)=4 m u_{x}+2 m_{x} u=P_{0} d K_{-6}
$$

that is, with $t_{-4}=t$,

$$
u_{t}-u_{x x t}=6 u_{x} u-4 u_{x x} u_{x}-2 u u_{x x x} .
$$

The conserved quantities $H_{i}$ and $K_{-i}$ commute with each other by the bi-Hamiltonian construction. Therefore we can collect the two iteration chain into a unique one defining the full $\mathrm{CH}$ 
hierarchy

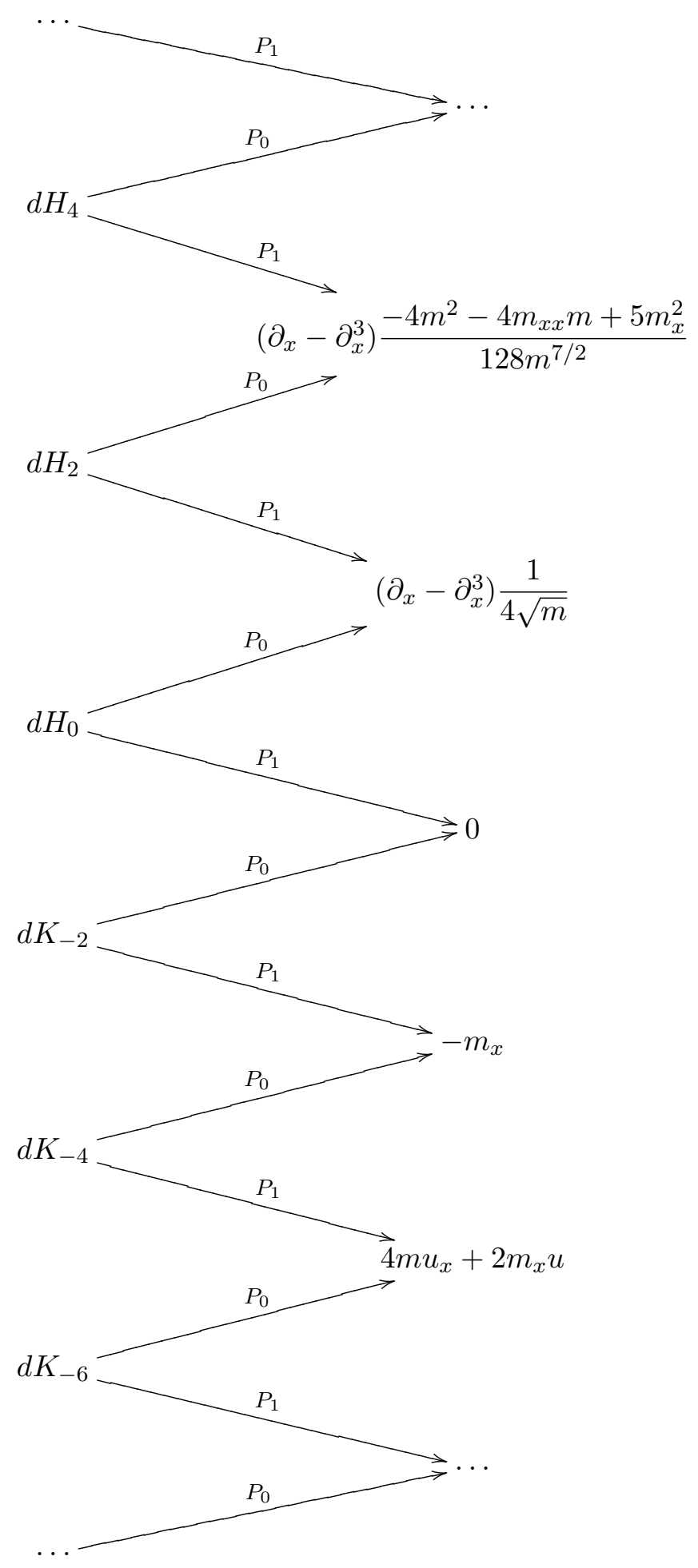

\section{The Noether currents}

In the $\mathrm{KdV}$ case the KP system has a simple interpretation as a generalization of the Noether relations (2) between conserved densities and related currents [13]. To understand if such a construction is still possible, we write down these currents in our case. In the case of local conserved 
quantities the calculation is already performed in [3] and gives the result:

$$
J^{(s)}=\sum_{i=0}^{s}\left(-\frac{1}{2} \partial_{x} v_{i}\left(z^{s-i+2}\right)+v_{i}\left(z^{s-i+2} h\right)\right)=\frac{z^{s+3}}{2}+O(z), \quad s \geq 0 .
$$

With similar computations, using

$$
\partial_{-2 s} m=-P_{z^{2}} \sum_{j=1}^{s} z^{2 j-2 s-2} d K_{-2 j}
$$

and $w(z)=z d K(z)$, one can show that, in the nonlocal case, the currents are:

$$
J^{(-s)}=\sum_{j=1}^{s}\left(\frac{1}{2} \partial_{x} w_{-j}\left(z^{j-s-1}\right)-w_{-j}\left(z^{j-s-1} k\right)\right)=\frac{z^{-s-2}}{2}+O(1), \quad s \geq 1 .
$$

Although it seems to possess the same structure, these two families of currents are really different. The local currents, as in the $\mathrm{KdV}$ case, are elements of a particular space called $J_{+}$. It is the span on the periodic smooth functions of the Faà di Bruno polynomials

$$
h^{(n)}=\left(\partial_{x}+h\right)^{n} z^{2}, \quad n \geq 0 .
$$

It holds

Proposition 1. The currents $J^{(2 s)}$, with $s \geq 0$, are elements of $J_{+}$.

Proof. (See [3].) Thanks to the representation (8), it suffices to show that $z^{2 i}$ and $z^{2 i} h$ are elements of $J_{+}$for all $i \geq 1$. First of all, $z^{2}=h^{(0)} \in J_{+}$and $z^{2} h=h^{(1)} \in J_{+}$by definition of $J_{+}$. Moreover, the Riccati equation (6) multiplied by $z^{2}$,

$$
h^{(2)}=\frac{z^{2}}{4}+z^{4} m
$$

shows that $z^{4}=1 / m\left(h^{(2)}-(1 / 4) h^{(0)}\right) \in H_{h}$. Acting with $\left(\partial_{x}+h\right)^{n}$ on both sides of (10) we prove the statement.

For every $s \geq 0$, the current $J^{(s)}$ has a essential singularity in zero and a pole of order $s+3$ at infinity. The space which contains these currents has to be generated by Laurent series with similar characteristics. Actually, in the proof of the previous proposition, a fundamental role is played by the fact that the conserved density $h(z)$, which is the seed for the Faà di Bruno polynomials, diverges both at 0 (essential singularity) and at $\infty$ (simple pole).

For every $s<0$, the current $J^{(s)}$ has a essential singularity at infinity and a pole of order $-s-2$ at zero. However the related generator of conserved quantities $k(z)$ is regular at 0 . Therefore $k(z)$ cannot generate the analogue of $J_{+}$for the nonlocal currents by means of the previous method.

As we will see in the following, the existence of this space is a key ingredient for the construction of a KP-CH system.

\section{The KP-CH system}

Let us then concentrate on the local currents $J^{(s)}$. They can be characterized in a unique way by the following two properties:

$$
\text { 1) } J^{(s)}=\frac{1}{2} z^{s+3}+O(z), \quad \text { 2) } J^{(s)} \in J_{+} .
$$


These currents are not a basis of the $J_{+}$space. To obtain a basis one has to add the constant $z^{2}$ so that $J_{+}=\left\langle z^{2}, J^{(n)}\right\rangle_{n \geq 0}$. Assuming that $h$ is an arbitrary Laurent series of the form

$$
h(z)=h_{-1} z+\sum_{i=0}^{+\infty} \frac{h_{i}}{z^{i}},
$$

where the coefficients $h_{i}$ are not constrained by the Riccati equation, we can define the currents $J^{(s)}$, for all $s \geq 0$, imposing the two above-mentioned properties. We define the $s$-th equation of the local $\mathrm{KP}-\mathrm{CH}$ system [3] as

$$
\partial_{s} h=\partial_{x} J^{(s)}, \quad s \geq 0 .
$$

It is an evolution equation in an infinite number of fields given by the coefficients $h_{-1}, h_{0}, h_{1}$, $\ldots$ of $h$. The main difference between this system and the standard KP system [17] is that the density $h$ is not an element of the space $J_{+}$. Nevertheless the equations (11) can be written using only elements of $J_{+}$

$$
\partial_{s} h^{(1)}=z^{2} \partial_{x} J^{(s)} .
$$

These equations are nonlinear in the space $J_{+}$because they involve the product of two elements of $J_{+}$. This nonlinearity in another difference between KP-CH and standard KP.

Directly from the equations of motion we easily see that this system possesses an infinite number of conserved quantities. Their densities are all the coefficients $h_{i}$ of $h(z)$.

It is well known that the KP equation in $2+1 \mathrm{D}$ can be obtained simply combining in a suitable way the first equation of the KP system in infinite fields. The analogue of the $2+1$ dimensional $\mathrm{KP}$ equation can be obtained also in our case. The smallest closed differential subsystem of evolution equation involves the first 5 fields $h_{-1}, \ldots, h_{3}$

$$
\begin{aligned}
& \partial_{0} h_{-1}=\partial_{x} \frac{h_{1}}{2 h_{-1}}, \quad \partial_{0} h_{0}=\partial_{x} \frac{h_{2}}{2 h_{-1}}, \quad \partial_{0} h_{1}=\partial_{x} \frac{h_{3}}{2 h_{-1}}, \\
& \partial_{1} h_{-1}=\partial_{x}\left(\frac{h_{2}}{h_{-1}}+\frac{1}{h_{-1}} \partial_{x} \frac{h_{1}}{2 h_{-1}}\right), \\
& \partial_{1} h_{0}=\partial_{x}\left(\frac{h_{3}}{h_{-1}}+\frac{h_{1}^{2}}{2 h_{-1}^{2}}+\frac{1}{h_{-1}} \partial_{x} \frac{h_{2}}{2 h_{-1}}\right) .
\end{aligned}
$$

After some simple algebraic manipulation one reduces this system to

$$
\begin{aligned}
& 2 u_{t}=\left(\frac{w}{u}\right)_{x}, \\
& u_{y}=2 v_{t}+\left(\frac{u_{t}}{u}\right)_{x}, \\
& v_{y}=2 w_{t}+2 \frac{w}{u} u_{t}+\left(\frac{v_{t}}{u}\right)_{x},
\end{aligned}
$$

where $h_{-1}=u, h_{0}=v, h_{1}=w$ and $t_{0}=t, t_{1}=y$.

\subsection{The evolution of the currents}

We study now the evolution of the currents $J^{(s)}$. In the standard KP case they are related to the Sato linear flows on the Grassmannian [13]. For the KP-CH equation the situation is somewhat different. By definition, the space $J_{+}$is invariant w.r.t. $\partial_{x}+h$ but the invariance is lost w.r.t. $\partial_{s}+J^{(s)}$. Actually the action of $\partial_{s}+J^{(s)}$ of the generic element of the basis of $J_{+}$is

$$
\left(\partial_{s}+J^{(s)}\right) h^{(k)}=\left(\partial_{s}+J^{(s)}\right)\left(\partial_{x}+h\right)^{k} z^{2}=z^{2}\left(\partial_{x}+h\right)^{k}\left(\partial_{s}+J^{(s)}\right) \subset z^{2} J_{+} .
$$


Remark 1. As we have seen in the introduction, the analogue for $\mathrm{KP}$ of the space $J_{+}$is the space $H_{+}$. The main difference between these two spaces is that in the KP-CH case the space $J_{+}$ is not invariant under the action of the operators $\left(\partial_{s}+J^{(s)}\right)$.

Using arguments similar to those used in [13] it is easy to show, for example in the case $z^{2} J_{+} \subset J_{+}$studied in the next paragraph, the commutativity of the flows generated by $\left\{\partial_{s}\right\}_{s} \geq 0$. Therefore, in the same case, we can introduce the function $\psi$ defined by $\partial_{s} \ln \psi=J^{(s)}$ and by $\partial_{x} \ln \psi=h$. Such a function exist also in the KP case and, in that case [24], it is the Baker-Akhiezer function.

We conjecture that the generalizations of $\psi$ are the Baker-Akhiezer functions for the integrable reductions of $\mathrm{KP}-\mathrm{CH}$.

\section{On the $\mathrm{KP}-\mathrm{CH}$ reductions}

To recover the local $\mathrm{CH}$ hierarchy from (11), one has to impose on $h$ the constraint given by the Riccati equation (6). Therefore all the fields $h_{i}$ can be written in terms of $m$ and its $x$ derivatives. Thus the local KP-CH system (11) reduces to the local $\mathrm{CH}$ hierarchy. As in the Gelfand-Dikii cases this reduction is a stationary reduction because the Riccati equation imply $J^{(1)}=\frac{z^{4}}{2}$ and the triviality of the evolution along $t_{1}$. From Proposition 1 it also follows that all $t_{2 s+1}$ are stationary. However, contrarily to the $\mathrm{KdV}$ case, the constraint given by Riccati equation (6) is not equivalent to $J^{(1)}=\frac{z^{4}}{2}$. The local Camassa-Holm equation is a strange reduction of a system obtained in its turn as a reduction from the $\mathrm{KP}-\mathrm{CH}$. Under the constraint

$$
J^{(1)}=\frac{z^{4}}{2}
$$

the evolution w.r.t. the time $t_{0}$ becomes

$$
\begin{aligned}
\partial_{0} h_{-1}= & -\frac{\partial_{x}\left(h_{-1}\right) h_{1}}{2 h_{-1}{ }^{2}}+\frac{\partial_{x} h_{1}}{2 h_{-1}}, \\
\partial_{0} h_{0}= & -\frac{3}{4} \frac{\left(\partial_{x} h_{-1}\right)^{2} h_{1}}{h_{-1}}+\frac{3}{4} \frac{\left(\partial_{x} h_{-1}\right)\left(\partial_{x} h_{1}\right)}{h_{-1}}+\frac{1}{4} \frac{\left(\partial_{x}^{2} h_{-1}\right) h_{1}}{h_{-1}}-\frac{1}{4} \frac{\partial_{x}^{2} h_{1}}{h_{-1}{ }^{2}} \\
\partial_{0} h_{1}= & \frac{\left(\partial_{x} h_{-1}\right) h_{1}^{2}}{2 h_{-1}{ }^{3}}-\frac{15}{8} \frac{h_{1}\left(\partial_{x} h_{-1}\right)^{3}}{h_{-1}}+\frac{15}{8} \frac{\left(\partial_{x} h_{-1}\right)^{2} \partial_{x} h_{1}}{h_{-1}}+\frac{5}{4} \frac{h_{1}\left(\partial_{x}^{2} h_{-1}\right) \partial_{x} h_{-1}}{h_{-1}{ }^{5}} \\
& -\frac{3}{4} \frac{\left(\partial_{x} h_{-1}\right) \partial_{x}^{2} h_{1}}{h_{-1}}-\frac{h_{1} \partial_{x} h_{1}}{2 h_{-1}{ }^{2}}-\frac{\left(\partial_{x}^{2} h_{-1}\right) \partial_{x} h_{1}}{2 h_{-1}}-\frac{h_{1} \partial_{x}^{3} h_{-1}}{8 h_{-1}}+\frac{\partial_{x}^{3} h_{1}}{8 h_{-1}{ }^{3}} .
\end{aligned}
$$

One can see that the field $h_{0}$ does not affect the evolution of the system. This is true also for the following times of the reduction because the currents themselves do not depend on $h_{0}$. Actually, by direct computation, we can show that the current $J^{(0)}$ do not depend on $h_{0}$. By (12) all the successive currents do not depend on $h_{0}$.

The more compact way to write the system is

$$
\partial_{0} \alpha=D_{\alpha} \frac{\gamma}{\alpha}, \quad \partial_{0} \beta=-D_{\alpha}^{2} \frac{\gamma}{\alpha}, \quad \partial_{0} \gamma=\alpha D_{\alpha}^{3} \frac{\gamma}{\alpha},
$$

where $\alpha=h_{-1}, \gamma=h_{-1} h_{1}, \beta=h_{0}$ and $D_{\alpha}=\partial_{x} \cdot(1 / 2 \alpha)$.

The conserved densities for (15) are given by the condition (13) written in terms of the generator $h$ of the Faà di Bruno polynomials (9):

$$
\frac{1}{2 \alpha^{2}} h^{(2)}-\left(\frac{\alpha_{x}}{2 \alpha^{3}}+\frac{\beta}{\alpha^{2}}\right) h^{(1)}+\left(\frac{\beta_{x}}{2 \alpha^{2}}+\frac{\gamma}{\alpha^{2}}-\frac{\beta \alpha_{x}}{2 \alpha^{3}}-\frac{\beta^{2}}{\alpha^{2}}\right) h^{(0)}=\frac{z^{4}}{2} .
$$


The local $\mathrm{CH}$ can be obtained from this equation system under the constraint:

$$
\gamma=\frac{m_{x x}}{8 m}-\frac{5 m_{x}^{2}}{32 m^{2}}+\frac{1}{8}, \quad \beta=-\partial_{x} \ln \left(m^{1 / 4}\right), \quad \alpha=\sqrt{m}
$$

which reduces $(15)$ to $(7)$, i.e.:

$$
\partial_{0} m=\frac{1}{4}\left(\partial_{x}-\partial_{x}^{3}\right) \frac{1}{\sqrt{m}} .
$$

The same reduction transforms (16) into (6). An important property of (15) is related to its associated dispersionless system:

$$
\partial_{0} \alpha=\frac{1}{2} \partial_{x} \frac{y}{\alpha^{2}}, \quad \partial_{0} \beta=0, \quad \partial_{0} \gamma=0 .
$$

This trivial system is bi-Hamiltonian w.r.t. the Poisson tensors:

$$
P_{1}^{d}=\frac{1}{2}\left(\begin{array}{ccc}
\partial_{x} & 0 & 0 \\
0 & 0 & 0 \\
0 & 0 & 0
\end{array}\right), \quad P_{0}^{d}=\frac{1}{2}\left(\begin{array}{ccc}
0 & 0 & \partial_{x} \alpha \\
0 & 0 & 0 \\
\alpha \partial_{x} & 0 & \gamma \partial_{x}+\partial_{x} \gamma
\end{array}\right)
$$

and the Hamiltonians $H_{1}=-\int(\gamma / \alpha) d x$ and $H_{0}=\int\left(\gamma^{2} / 2 \alpha^{3}\right) d x$. The metric associated to $P_{1}^{d}$ is obviously degenerate, then this dispersionless system is not of "Dubrovin-Novikov type" [9]. This is an important difference between this reduction and the KdV equation because it is not possible to include that system into the Dubrovin's classification scheme.

\section{Conclusion}

In this paper we have studied the KP system related to the Camassa-Holm hierarchy [3]. This system seems very different from the standard KP case. The first difference is in the fact that the evolution of the currents (12) is not contained in the space $J_{+}$which plays the role of the projection on the positive part of the Sato Grassmannian naturally defined by the currents.

The second difference is that the first reduction, obtained fixing the first current, is not an integrable system whose dispersionless part is related to a non-degenerate metric. This could be seen as a motivation why they do not enter in the Dubrovin classification scheme [10].

In the paper we have defined, in a restricted case, a function $\psi$, and we conjecture that its generalizations are the Baker-Akhiezer functions for the $\mathrm{KP}-\mathrm{CH}$ integrable reductions. We are studying also whether in our case $\psi$ is an eigenfunction of a suitable Lax operator which can be used to construct a zero curvature equation. The open question that we will address is as follows: Are the local symmetries of $\mathrm{CH}$ bilinear?

\section{Acknowledgements}

The author thanks Marco Pedroni and Gregorio Falqui for very useful discussions and hints, and two anonymous referees for useful comments and bibliographical references.

\section{References}

[1] Aratyn H., van de Leur J., Clifford algebra derivations of tau functions for two-dimensional integrable models with positive and negative flows, SIGMA 3 (2007), 020, 29 pages, nlin.SI/0605027.

[2] Camassa R., Holm D.D., An integrable shallow water equation with peaked solitons, Phys. Rev. Lett. 71 (1993), 1661-1664, patt-sol/9305002. 
[3] Casati P., Lorenzoni P., Ortenzi G., Pedroni M., On the local and nonlocal Camassa-Holm hierarchies, J. Math. Phys. 46 (2005), 042704, 8 pages.

[4] Chen M., Liu S., Zhang Y., A two-component generalization of the Camassa-Holm equation and its solutions, Lett. Math. Phys. 75 (2006), 1-15, nlin.SI/0501028.

[5] Constantin A., On the inverse spectral problem for the Camassa-Holm equation, J. Funct. Anal. 155 (1998), 352-363.

[6] Clarkson P.A., Gordoa P.R., Pickering A., Multicomponent equations associated to non-isospectral scattering problems, Inverse Problems 13 (1997), 1463-1476.

[7] Constantin A., McKean H.P., A shallow water equation on the circle, Comm. Pure Appl. Math. 52 (1999), 949-982.

[8] Date E., Jimbo M., Kashiwara M., Miwa T., Transformation groups for soliton equations, in Nonlinear Integrable Systems, Editors M. Jimbo and T. Miwa, World Scientific, Singapore, 1983.

[9] Dubrovin B.A., Novikov S.P., Hydrodynamics of weakly deformed soliton lattices. Differential geometry and Hamiltonian theory, Uspekhi Mat. Nauk 44 (1989), no. 6, 29-98 (English transl.: Russian Math. Surveys 44 (1989), no. 6, 35-124).

[10] Dubrovin B., Zhang Y., Normal forms of hierarchies of integrable PDEs, Frobenius manifolds and GromovWitten invariants, math.DG/0108160.

[11] Estevez P.G., Prada J., Hodograph transformations for a Camassa-Holm hierarchy in $2+1$ dimensions, J. Phys. A: Math. Gen. 38 (2005), 1287-1297, nlin.SI/0412019.

[12] Falqui G., On a Camassa-Holm type equation with two dependent variables, J. Phys. A: Math. Gen. 39 (2006), 327-342, nlin.SI/0505059.

[13] Falqui G., Magri F., Pedroni M., Bihamiltonian geometry, Darboux coverings and linearization of the KP hierarchy, Comm. Math. Phys. 197 (1998), 303-324, solv-int/9806002.

[14] Fontanelli L., Lorenzoni P., Pedroni M., A 3-component extension of the Camassa-Holm hierarchy, nlin.SI/0604053.

[15] Ivanov R., Extended Camassa-Holm hierarchy and conserved quantities, Z. Naturforschung A 61 (2006), 133-138, nlin.SI/0601066.

[16] Khesin B., Misiolek G., Euler equations on homogeneous spaces and Virasoro orbits, Adv. Math. 176 (2003), 116-144, math.SG/0210397.

[17] Konopelchenko B., Martinez Alonso L., Medina E., Singular sector of the Kadomtsev-Petviashvili hierarchy, $\bar{\partial}$ operators of nonzero index, and associated integrable systems, J. Math. Phys. 41 (2000), 385-413, solv-int/9806001.

[18] Kraenkel R.A., Senthilvelan M., Zenchuk A.I., Lie symmetry analysis and reductions of a two-dimensional integrable generalization of the Camassa-Holm equation, Phys. Lett. A 273 (2000), 183-193.

[19] Lenells J., Conservation laws of the Camassa-Holm equation, J. Phys. A: Math. Gen. 38 (2005), 869-880.

[20] Lorenzoni P., Pedroni M., On the bi-Hamiltonian structures of the Camassa-Holm and Harry Dym equations, Int. Math. Res. Not. 75 (2004), 4019-4029, nlin.SI/0407057.

[21] Martinez Alonso L., Shabat A.B., On the prolongation of a hierarchy of hydrodynamic chains, New trends in integrability and partial solvability, NATO Sci. Ser. II Math. Phys. Chem., Vol. 132, Kluwer Acad. Publ., Dordrecht, 2004, 263-280.

[22] Martines Alonso L., Shabat A.B., Hydrodynamic reductions and solutions of the universal hierarchy, Teoret. Mat. Fiz. 140 (2004), 216-229 (English transl.: Theoret. and Math. Phys. 140 (2004), 1073-1085), nlin.SI/0312043.

[23] Magri F., Casati P., Falqui G., Pedroni M., Eight lectures on integrable systems, in Integrability of Nonlinear Systems, Lecture Notes in Physics, Vol. 638, Editors Y. Kosmann-Schwarzbach et al., 2004, 209-250.

[24] Casati P., Falqui G., Magri F., Pedroni M., Soliton equations, bi-Hamiltonian manifolds and integrability, $21^{\circ}$ Coloquio Brasileiro de Matemetica (21st Brazilian Mathematics Colloquium), Instituto de Matemetica Pura e Aplicada (IMPA), Rio de Janeiro, 1997.

[25] Reyes E.G., Geometric integrability of the Camassa-Holm equation, Lett. Math. Phys. 59 (2002), $117-131$.

[26] Shabat A., Universal solitonic hierarchy, J. Nonlinear Math. Phys. 12 (2005), suppl. 1, 614-624.

[27] Sato M., Sato Y., Soliton equations as dynamical systems on infinite-dimensional Grassmann manifold, in Nonlinear PDEs in Applied Sciences (US-Japan Seminar, Tokyo), Editors P. Lax and H. Fujita, NorthHolland, Amsterdam, 1982, 259-271.

[28] Segal G., Wilson G., Loop groups and equations of the KdV type, Publ. Math. IHES 61 (1985), 5-65. 